\title{
THE STRUCTURE OF CANCELLATIVE POWER-FREE GROUPS
}

\author{
by A. GEDDES and R. G. WALKER \\ (Received 15 January, 1966)
}

1. Introduction. The definition of a power-free group will be found in [1]. It is a partial algebraic system which, roughly speaking, may be thought of as a group in which (with the exception of the identity) squares and higher powers of an element are not defined.

It has been shown [1, Theorem 3.3] that the usual cancellation laws need not hold in a power-free group. When these laws do hold, the power-free group is called cancellative. In this paper we shall be solely concerned with cancellative power-free groups and the term 'power-free group' is to be understood to mean 'cancellative power-free group'.

A power-free group can be obtained by taking any group which has no elements of order 2 and simply omitting squares, and one which arises in this way is called complete; otherwise it is said to be incomplete. As was shown in [1, Theorem 4.3], there is at least one incomplete power-free group, namely the quaternion power-free group. This is obtained from the ordinary quaternion group by removing the element of order 2 and, in the set consisting of the remaining seven elements, using the same composition law as that of the quaternion group but omitting the definition of squares. Thus, although the quaternion power-free group is not complete, it can be embedded in a group. It is therefore natural to ask if every power-free group can be embedded in a group. This was the basic question considered in [1], and we are now able to answer it in the affirmative. However, in the present paper, we shall prove more: we shall show that the quaternion power-free group is the only incomplete power-free group, i.e. that, apart from the quaternion power-free group, any power-free group can be obtained from a suitable ordinary group by the omission of squares.

2. Preliminary results. It was noted in [1] that there is only one power-free group of order 3 (necessarily complete), and hence we shall assume in what follows that any powerfree group considered has more than three elements.

We saw in [1, Theorem 5.4] that, in testing for completeness, we have to consider expressions of the type $a(a b)$; this is the motivation behind the lemma which follows.

LEMMA 2.1. Let $a$ and $b$ be elements of $a$ power-free group $P$ such that $a$ is irregular and $b \neq e, a, a^{-1}$. Then

Further,

$$
\text { either } a(a b)=a^{-1} b \text { or } a(a b)=b^{-1} \text {. }
$$

$$
\text { either }(b a) a=b a^{-1} \text { or } \quad(b a) a=b^{-1} \text {. }
$$

Proof. Let us suppose that $a(a b) \neq b^{-1}$.

We first show that $(a(a b)) b^{-1} \neq b$. Assuming the contrary, we have $(a(a b)) b^{-1}=b$, so that $b$ is regular with $s_{b}=a(a b)$. Hence, since $a$ is irregular, we find that $a b=a^{-1} s_{b}$. It now follows from [1, Lemma 5.1] that $a b=\left(a^{-1} b\right) b$, and, on cancelling $b$, we obtain $a=a^{-1} b$, which contradicts the irregularity of $a$. 
Consequently we can find $x \in P$, namely $x=(a(a b)) b^{-1}$, such that

Hence, since $a$ is irregular,

$$
a(a b)=x b \text {. }
$$

$$
a b=a^{-1}(x b) .
$$

Let us now further assume that $x \neq a^{-1}$ and that $a^{-1} x \neq b$; then relation (2) gives

$$
a b=\left(a^{-1} x\right) b \text {. }
$$

Accordingly, by cancellation, we obtain $a=a^{-1} x$, which implies that $a$ is regular, contrary to hypothesis.

We conclude, therefore, that, if $a(a b) \neq b^{-1}$, then either $x=a^{-1}$ or $a^{-1} x=b$.

Suppose that $a^{-1} x=b$; then, since $a \neq b$, we have $x=a b$, so that from the relation (1) we obtain

$$
a(a b)=(a b) b
$$

Let $a b=c$; then, by the irregularity of $a$, we have $c \neq a^{-1}$ and (3) gives

$$
\begin{aligned}
a c & =c b \\
& =c\left(a^{-1} c\right) \\
& =\left(c a^{-1}\right) c \quad(\text { since } a \neq e) .
\end{aligned}
$$

This implies, by cancellation, that $a=c a^{-1}$, contrary to our hypothesis that $a$ is irregular.

We have thus shown that, if $a(a b) \neq b^{-1}$, then $x=a^{-1}$, i.e. that $a(a b)=a^{-1} b$, as required.

To establish the second part of the lemma we note that the irregularity of $a$ implies that of $a^{-1}$. Hence, by the first part, if $b^{-1} \neq e, a^{-1}, a$, i.e. if $b \neq e, a, a^{-1}$, then

$$
\text { either } a^{-1}\left(a^{-1} b^{-1}\right)=a b^{-1} \text { or } a^{-1}\left(a^{-1} b^{-1}\right)=b \text {. }
$$

The result now follows by inverting these relations.

An element $a$ of a power-free group $P$ is irregular if and only if its inverse $a^{-1}$ is irregular. Hence the irregular elements occur in pairs. If $a$ and $b$ are irregular elements of $P$ and if $b \neq a$ and $b \neq a^{-1}$, we shall say that $a$ and $b$ are distinct irregular elements.

LEMMA 2.2. Let $a$ and $b$ be distinct irregular elements of $a$ power-free group such that

then

$$
a(a b)=a^{-1} b
$$

$$
\text { (i) } b(b a)=b^{-1} a, \text { (ii) } a\left(a b^{-1}\right)=a^{-1} b^{-1} \text {. }
$$

Proof. (i) By Lemma 2.1,

$$
\text { either } b(b a)=b^{-1} a \text { or } b(b a)=a^{-1} \text {. }
$$

Assume that the latter holds; then, since $b \neq a$, we have $b a=b^{-1} a^{-1}$. Hence, taking inverses, we find that $a b=a^{-1} b^{-1}$; consequently $a(a b)=b^{-1}$. If we combine this with the given relation, we obtain the contradiction that $b$ is regular, and from this (i) follows. 
(ii) Again, by Lemma 2.1, if the stated result does not hold, then $a\left(a b^{-1}\right)=b$, and from this we obtain $a b^{-1}=a^{-1} b$. However, $a^{-1} b=a(a b)$ (given); hence, using cancellation, we have $a b=b^{-1}$, giving again the contradiction that $b$ is regular.

Lemma 2.3. Let $a$ and $b$ be elements of $a$ power-free group such that $b \neq e, a$. Suppose that $a$ is irregular and that $a(a b)=b^{-1}$; then $b$ is also irregular.

Proof. From the relation $a(a b)=b^{-1}$ it follows, since $a \neq b$, that $a b=a^{-1} b^{-1}$, and from this we obtain $b a=b^{-1} a^{-1}$ by inverting. Accordingly, since $a \neq e$, so that $b \neq b a$, we have

$$
b(b a)=b\left(b^{-1} a^{-1}\right)=a^{-1} .
$$

If we assume that $b$ is regular, then this gives [1, Lemma 5.1]

$$
s_{b} a=a^{-1},
$$

which implies that $a$ is regular. Hence $b$ is irregular.

COROLLARY 2.4. If $a$ is an irregular element of a power-free group and $b$ is a regular element different from $e$, then

Proof. By Lemma 2.1,

$$
a(a b)=a^{-1} b .
$$

$$
\text { either } a(a b)=a^{-1} b \quad \text { or } \quad a(a b)=b^{-1} \text {. }
$$

If the second of these holds, then (Lemma 2.3) $b$ is irregular, which is not the case. Consequently the first holds, as asserted.

COROLLARY 2.5. Any power-free group with precisely two irregular elements is complete.

Proof. Let $a$ be any irregular element; then the two irregular elements are $a$ and $a^{-1}$. If $b$ is any element different from $e, a, a^{-1}$, then $b$ is necessarily regular and, by Corollary 2.4 , we have

and similarly

$$
a(a b)=a^{-1} b ;
$$

$$
a^{-1}\left(a^{-1} b\right)=a b \text {. }
$$

Accordingly, by [1, Theorem 5.4], the power-free group is complete.

If we combine this result with the fact [1, Corollary 5.5] that any power-free group in which every element is regular is complete, it follows that in the sequel we need only consider power-free groups having at least two distinct irregular elements. We recall (Lemma 2.1) that if $u$ and $v$ are two such elements, then

$$
\text { either } u(u v)=u^{-1} v \text { or } u(u v)=v^{-1} \text {. }
$$

We shall see that the structure of the power-free group is determined by the one of these which holds. 


\section{The structure theorem.}

LemMa 3.1. Let $P$ be a power-free group containing two distinct irregular elements $u$ and $v$ such that

$$
u(u v)=u^{-1} v
$$

then $P$ is complete.

Proof. Let $a$ be any irregular element of $P$ and $b$ any element different from $e, a, a^{-1}$. Then $\left[1\right.$, Theorem 5.4] the result will follow if we show that $a(a b)=a^{-1} b$.

We divide the proof into two parts.

Part 1. Here we consider the particular case in which $a=u$, and show that

$$
u(u b)=u^{-1} b \text { for all irregular } b \neq u, u^{-1} \text {. }
$$

We may assume that $b \neq v$ and $b \neq v^{-1}$, for the case in which $b=v$ follows from (4), while that in which $b=v^{-1}$ follows from (4) and Lemma 2.2, (ii).

Consider now the following equations in the unknowns $x_{1}, x_{2}$ :

We shall write these in the form

$$
b=v x_{1} \text { and } b=v^{-1} x_{2} .
$$

$$
b=v_{1} x_{1} \text { and } b=v_{2} x_{2} \text {, }
$$

where $v_{1}=v, v_{2}=v^{-1}$. Since, for $i=1,2$, the element $v_{i}$ is irregular and $b \neq v_{i}$, it follows that these equations are both soluble.

Now the four products $u v_{i}, u^{-1} v_{i}(i=1,2)$ all exist. Let us assume for the present that, for one of the two values of $i$,

$$
x_{i} \neq u v_{i} \text { and } x_{i} \neq u^{-1} v_{i}
$$

Then, for this value of $i$, we have

$$
u(u b)=u\left(u\left(v_{i} x_{i}\right)\right)=u\left(\left(u v_{i}\right) x_{i}\right), \quad \text { since } u \neq v_{i} \text { and } u v_{i} \neq x_{i} .
$$

From (4) and the fact (noted above) that it also holds when $v$ is replaced by $v^{-1}$, it follows that

$$
u\left(u v_{i}\right)=u^{-1} v_{i} \quad(i=1,2)
$$

Accordingly, for the above $i$,

i.e.

$$
u(u b)=\left(u\left(u v_{i}\right)\right) x_{i}, \quad \text { since } v_{i} \neq e \text { and } u^{-1} v_{i} \neq x_{i},
$$

Hence

$$
u(u b)=\left(u^{-1} v_{i}\right) x_{i} .
$$

so that

$$
u(u b)=u^{-1}\left(v_{i} x_{i}\right), \text { since } v_{i} x_{i}=b \neq u^{-1},
$$

$$
u(u b)=u^{-1} b
$$

Thus Part 1 will follow if we show that, for at least one value of $i$, we have both $x_{i} \neq u v_{i}$ and $x_{i} \neq u^{-1} v_{i}$.

If $x_{1} \neq u v_{1}$ and $x_{1} \neq u^{-1} v_{1}$, there is nothing further to prove. Consequently we suppose that either $x_{1}=u v_{1}$ or $x_{1}=u^{-1} v_{1}$. Let us take the first of these. We shall show that the result will go through with $i=2$, i.e. that $x_{2} \neq u v_{2}$ and $x_{2} \neq u^{-1} v_{2}$. 
Assuming the contrary, we have to consider two cases.

Case (i): $x_{1}=u v_{1}$ and $x_{2}=u v_{2}$. Here we have

and

$$
b=v_{1} x_{1}=v_{1}\left(u v_{1}\right)=v(u v)
$$

so that

$$
\begin{gathered}
b=v_{2} x_{2}=v_{2}\left(u v_{2}\right)=v^{-1}\left(u v^{-1}\right), \\
v(u v)=v^{-1}\left(u v^{-1}\right) .
\end{gathered}
$$

Hence, using the irregularity of $v$, we obtain

It now follows from Lemma 2.1 that

$$
u v=v^{-1}\left(v^{-1}\left(u v^{-1}\right)\right)
$$

either

$$
\begin{aligned}
& u v=v\left(u v^{-1}\right) \\
& u v=\left(u v^{-1}\right)^{-1} .
\end{aligned}
$$

If (5) holds, then $u v=(v u) v^{-1}$; hence

If the first of these applies, then

$$
\begin{aligned}
v u & =(u v) v \\
& =u v^{-1} \text { or } u^{-1}(\text { Lemma } 2.1)
\end{aligned}
$$

$$
b=v_{1} x_{1}=v(u v)=(v u) v=\left(u v^{-1}\right) v=u,
$$

while, if the second applies, $u$ must be regular. Since neither of these is the case, (5) cannot hold.

Suppose now that (6) holds; then

$$
\begin{aligned}
b & =v_{1} x_{1}=v(u v)=v\left(u v^{-1}\right)^{-1} \\
& =v\left(v u^{-1}\right) \\
& =v^{-1} u^{-1} \text { or } u \quad(\text { Lemma 2.1). }
\end{aligned}
$$

If the first applies, then $b=(u v)^{-1}$, so that from $b=v(u v)$ we obtain $b=v b^{-1}$, which implies that $b$ is regular. Since $b$ is neither regular nor equal to $u$, it follows that (6) cannot hold.

Accordingly if $x_{1}=u v_{1}$, then $x_{2} \neq u v_{2}$.

Case (ii): $x_{1}=u v_{1}$ and $x_{2}=u^{-1} v_{2}$. In this case

and

$$
b=v_{1} x_{1}=v_{1}\left(u v_{1}\right)=v(u v)
$$

Hence

$$
b=v_{2} x_{2}=v_{2}\left(u^{-1} v_{2}\right)=v^{-1}\left(u^{-1} v^{-1}\right) \text {. }
$$

so that $v(u v)=e$, which gives $u v=v^{-1}$. Thus we obtain the contradiction that $v$ is regular, and this shows that Case (ii) cannot apply.

The remaining two cases, namely

02

$$
x_{1}=u^{-1} v_{1}, x_{2}=u v_{2} \text { and } x_{1}=u^{-1} v_{1}, x_{2}=u^{-1} v_{2}
$$


can be treated in exactly the same way as the above, the first corresponding to Case (ii) and the second to Case (i).

This completes the proof of Part 1.

Part 2. We establish here the general result.

We have shown that

$$
u(u b)=u^{-1} b \text { for all irregular } b \neq u, u^{-1} .
$$

Hence, for all such $b$, we have, by Lemma 2.2, (i),

$$
b(b u)=b^{-1} u .
$$

If we take a $b$ satisfying (7), then we see that we have a situation exactly the same as that with which we started in Part 1. Accordingly, by the result of Part 1 , we have, for any irregular $a \neq b, b^{-1}$ with $b$ irregular and $b \neq u, u^{-1}$,

$$
b(b a)=b^{-1} a,
$$

i.e. $\quad b(b a)=b^{-1} a$ for all irregular $b \neq a, a^{-1}, u, u^{-1}$.

If we now apply Lemma 2.2, (i), we obtain

$$
a(a b)=a^{-1} b \text { for all irregular } b \neq a, a^{-1}, u, u^{-1} .
$$

We now observe that, by Corollary 2.4, this result remains true if $b$ is regular and different from $e$. Further, it also holds in the cases $b=u$ and $b=u^{-1}$, as can be seen by replacing $b$ by $a$ in (7) and then replacing $u$ by $u^{-1}$ in the resulting equality (using Lemma 2.2, (ii)). We have thus shown that

as required.

$$
a(a b)=a^{-1} b \text { for all } b \neq e, a, a^{-1},
$$

This lemma shows that as soon as a power-free group $P$ has two distinct irregular elements satisfying the condition (4), it is complete. Hence, by Lemma 2.1, it only remains for us to consider the case in which $P$ has at least two distinct irregular elements and any two such elements $a$ and $b$ satisfy the condition

$$
a(a b)=b^{-1} .
$$

Lemma 3.2. Let $P$ be a power-free group with at least two distinct irregular elements, and suppose that any two distinct irregular elements $a$ and $b$ satisfy the condition

$$
a(a b)=b^{-1} \text {. }
$$

Then $P$ has no regular elements other than $e$.

Proof. We first show that the product $a b$ of any two distinct irregular elements $a$ and $b$ is also an irregular element. Assume the contrary; then, since $a b$ is regular and $a$ is irregular and since $a b \neq e$, we have, by Corollary 2.4,

i.e.

$$
\begin{aligned}
& a(a(a b))=a^{-1}(a b), \\
& a(a(a b))=b .
\end{aligned}
$$


Accordingly, by (8), $a b^{-1}=b$; but this cannot be the case since $b$ is irregular. Hence $a b$ is irregular.

It follows from this result that the set $Q$ consisting of all the irregular elements together with $e$ forms a power-free subgroup of $P$. Hence, if $a$ and $b$ are as above and if $c=a b$, then we see, using the irregularity of $a$ and $b$, that $Q$ contains the seven distinct elements

$$
e, a, a^{-1}, b, b^{-1}, c, c^{-1} \text {. }
$$

Let $R=P-Q$; then what we have to show is that $R$ is empty. We shall assume that this is not the case and derive a contradiction.

Let $r \in R$ and let $a$ be an irregular element. Consider $r^{-1} a$; this is the product of a regular element and an irregular element and hence is a regular element, since $Q$ is closed. Thus we may write $r^{-1} a=s$, where $s \in R$ and where clearly $r^{-1} \neq s$. Now $r \neq s$, since otherwise we should have $a=s_{r} \in R$, for the regularity of $r$ implies that of $s_{r}$ [1, Lemma 5.2]. Hence we have

$$
r s=r\left(r^{-1} a\right)=a \in Q .
$$

Since, by the observation made above, $Q$ contains at least six irregular elements, it follows that we can choose an irregular element $t \in Q$ such that

$$
t \neq r s,(r s)^{-1}, r^{-1} s,\left(r^{-1} s\right)^{-1} .
$$

From that fact that $t \neq r s$, we obtain from $(8)$ that

$$
(r s) t=(r s)^{-1} t^{-1}
$$

hence

$$
((r s) t) t=(r s)^{-1},
$$

and consequently, since $t \neq s^{-1} r$, we have

$$
(r(s t)) t=(r s)^{-1} .
$$

Now it is easily deduced from Corollary 2.4 that $(s t) t=s t^{-1}$; further, by hypothesis, $t \neq r^{-1} s$. Hence $r\left(s t^{-1}\right)=(r s)^{-1}$. Finally, this may be written $(r s) t^{-1}=(r s)^{-1}$, since $t \neq(r s)^{-1}$. Accordingly $r s$ is regular, and this gives the required contradiction.

LEMMA 3.3. Let $P$ be a power-free group in which every element (other than e) is irregular, and suppose that, for all distinct irregular elements $x$ and $y$,

$$
x(x y)=y^{-1}
$$

then $P$ is the quaternion power-free group.

Proof. We first show that, for all distinct irregular $x$ and $y$,

$$
(x y) x=y \text {. }
$$

From (9) it follows that

$$
x y=x^{-1} y^{-1}
$$

and hence

$$
(x y) x=\left(x^{-1} y^{-1}\right) x .
$$

Now apply (11) to the elements $x^{-1} y^{-1}$ and $x$ to obtain

accordingly

$$
\begin{aligned}
& (x y) x=\left(x^{-1} y^{-1}\right)^{-1} x^{-1} ; \\
& (x y) x=(y x) x^{-1}=y .
\end{aligned}
$$


Let $a$ and $b$ be distinct irregular elements and set $a b=c$. Then, as was noted in the last lemma, the seven elements

$$
e, a, a^{-1}, b, b^{-1}, c, c^{-1}
$$

are distinct. Further, it can easily be deduced from (9), (10) and (11) that the subset $Q$ consisting of these seven elements is a power-free subgroup of $P$, which is in fact the quaternion power-free group. Thus the proof will be complete if we show that $Q=P$.

Assume that $Q \neq P$ and let $x \in P, x \notin Q$. We have

$$
(x a)(x b)=((x a) x) b,
$$

since $(x a) x=a$ (by (10)) and $a \neq b$; hence

$$
(x a)(x b)=a b=c .
$$

Also

$$
(x a)(b x)=((x a) b) x \text {, }
$$

since $x a \neq b,(x a) b=x(a b)=x c$ and $c \neq e$; hence

$$
\begin{aligned}
(x a)(b x) & =(x c) x \\
& =c, \text { by }(10) .
\end{aligned}
$$

Accordingly

$$
(x a)(x b)=(x a)(b x) \text {, }
$$

so that, by cancellation, $x b=b x$.

Hence, by (11), $x b=b^{-1} x^{-1}=(x b)^{-1}$, and this gives $x b=e$, i.e. $x=b^{-1} \in Q$, a contradiction.

THEOREM 3.4. The only incomplete power-free group $P$ is the quaternion power-free group.

Proof. $P$ cannot consist entirely of regular elements [1, Corollary 5.5] nor can it have precisely two irregular elements (Corollary 2.5). Further, the conditions of Lemma 3.1 cannot be satisfied, and hence (Lemma 2.1) those of Lemma 3.2 must be satisfied. The proof is now completed by applying Lemma 3.3.

\section{REFERENCE}

1. A. Geddes, Power-free groups, Proc. Cambridge Philos. Soc. 60 (1964), 393-408.

\section{UNIVERSITY OF GLASGOW}

GLASGOW, W.2 